Fig. 6. Durchschnitt durch einen kleinen Markraum. In der Mitte ist der Querschnitt eines Gefässes gelegen, das in einiger Entférnung von einèr lockeren Scheide umgeben ist. An diese schliessen sich verästigte Zellen an, deren Ausläufer ein geschlossenes Netz bilden. Die Räume zwischen diesen enthalten rundliche Zellen. In dem Netz anastomosirender Zellen ist Farbstoff abgeschieden, ebenso in den Knochenkanälchen und Knochenläcunen der innersten Lamelle.

\title{
III.
}

\section{Experimentelles über fettige Degeneration der Nierenepithelien.}

\author{
Von Dr. Otto von Platen, \\ Assistent am pathologisehen Institut zu Bonn.
}

Mit den Versuchen, welche man bisher angestellt hat, fettige Degeneration des Nierenepithels zu erzeugen, ist man nicht gerade glücklich gewesen. Wenigstens ist es nicht gelungen, die Ursachen dieser Ernährungsstörung bis auf einfache, so zu sagen elementare, Vorgänge zu verfolgen.

So schätzenswerth auch die leider so kurz ausgeführten Angaben yon $\mathrm{Mauasein}^{1}$ ) sind, dass die Inanition schliesslich zu fettiger Degeneration der Niere führt, so ist doch diese Bedingung gewiss eine so complicirte, geht mit solchen Umwälzungen im gesammten Stoffwechsel des Organismus einher, dass sie einen Rückschluss in Bezug auf das Zustandekommen der Nierenaffection nicht gestattet. Dies gilt noch mehr von der Phosphorvergiftung. Durch-

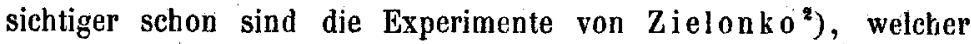
bei Verengerung der Aorta ascendens zuweilen fettige Degeneration des Nierenepithels erzeugte. Man sollte nun glauben, dass dies Ergebniss zu dem weiteren Versuch hătte einladen müssen, auf eine mehr directe. Weise das Verhältniss der arteriellen Zufuhr zu dem genannten Prozess klar zu legen. Statt dessen sehen wir die fer-

1) Med. Centralblatt. 1868. No. 18. S. 273.

2) Dieses Archiv Bd. 61. S. 267. 
neren Arbeiten fast nur damit beschäftigt, den Einfluss der gehinderten venösen Blutbewegung festzustellen. So haben Buchwald und Litten ${ }^{1}$ ) die Nierenvene unterbunden und durch Staung des Venenblutes in der That fettige Degeneration des Nierenepithels eintreten sehen. Jedoch widersprechen diesem Ergebniss in einer folgenden Abhandlung Weissgerber und Perls ${ }^{2}$ ): "Wenn wir die Nierenvene zubinden, werden wir nicht blos eine Verlangsamung der Blutströmung in der Niere mit längerem Verweilen kohlensäurereichen Blutes in derselben verursachen, wie's zum Begriffe der Stauung gehört, sondern wir werden geradezu einen Stillstand der Blutströmung verursachen; dem entspricht auch der Nierenbefund in unseren eben angefïhrten Experimenten. Die Niere zeigt geradezu grösstentheils die Beschaffenheit eines todten Blutkuchens, wie ein hämorrhagischer Infarct sie zeigt, mit jener Trockenheit, dem Feblen des normalen Gewebsglanzes u. s. w. Also haben wir es schon gar nicht mehr mit einer lebenden Niere zu thun etc." Dazu ist zu bemerken, dass auch Buchwald und Litten der Stauung des Venenblutes den beschriebenen Einfluss nicht vindiciren, sondern einer gleichzeitigen Behinderung des arteriellen Zuflusses als einer Folge der gewaltigen Blutstockung. Weissgerber und Perls suchten dann mehr dem Begriff der Staung zu entsprechen, nur eine Verlangsamung des Venenblutrückflusses zu verursachen: durch Verengerung der Vena renalis. Sie fanden „die Veränderungen der Epithelien löchst unbedeutend, in etwas Trübung und Quellung bestehend".

Dies sind inn Ganzen die Resultate der neueren Versuche über den Einfluss der Circulationsanomalien auf die Epithelien der Niere (abgesehen von einigen Arbeiten, welche sich mit gleichen Versuchen beschäftigten, z. B. mit Unterbindung der Nierenvene), und betrachtet man sie, so wird man, glaube ich, mit meinen Worten übereinstimmen, dass es nicht gelungen sei, die fettige Degeneration auf einfache Zustände zurückzuführen. Sollten dieselben nicht dennoch experimentell darzustellen sein?

Ich habe nun in der folgenden Arbeit den Weg, welchen Zielonko betreten hatte, weiter verfolgt, habe auf eine mebr di-

1) Dieses Archiv Bd. 66. S. 145.

2) Archiv für exper. Path. und Pharmak. Bd. VI. S. 113. 
recte Weise die Bedeutung der ungenügenden arteriellen Zufuhr für das Nierenparenchym klar zu legen versucht: nehmlich durch Verengerung der Arteria renalis.

Die gewonnenen Resultate könnte ich in zweierlei Hinsicht verwerthen: einmal für die Betrachtung der fettigen Degenerationen überhaupt, dann im Speciellen für die Betrachtung der krankhaften Prozesse in den Nieren. Beide Kapitel sind so gross, dass eine eingehende Auseinandersetzung zu geben, für den Augenblick mir nicht möglich ist.

Was die Operationsmethode angeht, so standen mir, um zur Arteria renalis zu gelangen, zwei Wege offen: vom Rücken des Tbieres aus extraperitoneal, oder von der Bauchböhle aus. Der erstere ist nothwendig, wenn- man das Thier längere Zeit am Leben erhalten will, leidet aber an dem Uebelstande, dass die Niere selbst mit dem operativen Eingriff mehr in Berührung kommt. Man dringt in den Muskelinterstitien in die Tiefe. Ist man an die Niere gelangt, so kann man in situ an der Arterie operiren: dann ist aber die Unführung eines Fadens oder Drahtes wegen der Tiefe der Wunde recht schwierig, zumal bei fettreichen Thieren. Auch kann es sich leicht ereignen, dass man nur einen Ast der Arterie statt der ganzen fasst: denn die Höbe der Theilung ist nicht ganz constant und man bekommt nur den einen Ast zu Gesicht, welcher den anderen deckt.

Stülpt man dagegen die Niere durch die Wunde vor, so kommt das Organ mit der Operation noch mehr in Berürung, ja ohne die nöthigen Schutzmittel gegen Kälte und Verdunstung kann man eine Ernährungsstörung erleben, welche sich als Necrose der äussersten Rindenschichten ausspricht. Alles dies fällt weg bei der Operation von der Bauchhöhle aus: man incidirt in der Linea alba oder in ihrer Năhe, nimmt vorquellende Darmschlingen heraus und sucht die Arterie in der Nähe der Wirbelsäule auf.

Die Verengerung der Arterie geschieht am besten mittelst eines Silberdrahtes, welchen man bis zur deullichen Abplattung der Arterie zusammendrückt. Schwierig ist es, beim lebenden Thier sich von der Durchgängigkeit der Arterie zu überzeugen. Nach dem Tode des Thieres habe ich es nicht unterlassen, mit den gewöhnlichen Vethoden: Versehiebung des Blutes über die verengte Stelle hinaus, oder Injection von Berliner-Blau-Lösung unter geringem 
Druck. Uebrigens kaun von Verschluss oder Thrombose überhaupt, glaube ich, garnicht die Rede sein: denn die Folge wäre Necrose des Organs, ein Zustand, an welchem schon nach einer Zeit von 24 Stunden, und zwar schon makroskopisch, nicbt zu zweifeln ist.

Es erübrigt jetzt noch, kurz des möglichen Einwandes zu gedenken, dass es sich bei den Resultaten um ein Kunstproduct gehandelt habe. Es ist dies, meiner Meinung nach, schon aus dem Grunde nicht anzunehmen, weil ich nach allen den dreien angegebenen Methoden operirt habe und in den gelungenen Fällen von genügender Verengerung auch fettige Degeneration nicht vermisst habe. Bei der Operation von der Bauchhöhle aus war die Niere ja überhaupt nicht dem operativen Eingriff ausgesetzt: und sollte man eine Operation in der Bauchböhle schon für genügend erachten, so kann ich erwidern, dass andere Operateure bei derselben Operation, nur an der Nierenvene, fettige Degeneration gerade nicht erzielten; ferner, dass ich bei gelungenen Versuchen wohl Peritonitis vermisst babe, aber nicht fettige Degeneration. Denn nur solche Versuche will ich als gelungen gelten lassen. Bei den Operationen vom Rücken aus habe ich mich durch Controlversuche ausdrücklich von der Richtigkeit meiner Kesultate überzeugt, habe den Draht um die Arterie geführt, ohne ihn fest zuzuschnüren. Den letzten Einwand endlich, dass die Operation zugleich mit der Verringerung des nöthigen Ernäbrungsmaterials zur Hervorbringung des Endeffectes concurrirt habe, den durch Controlversuche zu widerlegen, bin ich allerdings nicht in der Lage; verweise aber auf die Arbeit von Zielouko, welchen die Verengerung der Aorta ascendens zu demselben Resultat gefürt hatte.

Aus der Reihe meiner Versuche seien einjge Beispiele kurz angeführt:

\section{Versuch I.}

Einem mittelgrossen, gelben Kaninchen verengerte ich die linke Arteria renalis vom Rücken aus in situ auf die beschriebene Art. Die Operation ging verbältnissInässig schnell vor sich. In der folgenden Zeit befand sich das Thier vollkommen wohl. Nach 7 Tagen wurde es getödtet und sofort secirt. Die Wunde war ohne Eiterung vernarbt. Der Stamm der Nierenarterie mässig stark verengert. Das Blut äber die Stelle der Verengerung hinaus verschiebbar. Die linke Niere erscheint auf der Oberfläche blasser, zugleich weisslicher gefärbt: im Uebrigen dieselbe ganz glatt. Auf der Schnittfläche trat überall flüssiges Blut aus. Hier ebenfalls die Hindensubstanz deutlich blässer und trüber. Marksubstanz ziemlich blutreich. 
Die milkroskopische Untersuchung, am frischen Präparat vorgenommen, ergiebt: Schnitte der Rindensubstanz, sowohl von der Oberfläche, als von der Schnittläche genommen, zeigen in die Nierenepithelien eingelagert hellglänzende Kügelchen, glänzender und grösser, als die gewöhnlichen Albuminkörnchen. Zusatz von Essigsäure oder verdünnter Natronlauge liess sie noch stärker hervortreten, zeigte ihr Verbältniss za dem Zellkern als ein derartiges, dass sie nicht in ihm, wohl in naher Umgebung desselben, oft gruppenwels angehäuft erschienen, ohne zu confluiren. Darch Alkohol und Aether konnten sie extrahirt werden. Das interstitielle Gewebe völlig intact, ebenso die Glomernli. In der Marksubstanz fanden sich gleichfalls Fettkörnchen. Die rechte Niere von normalem Aussehen, blutreich: ibre Tubuli contorti zeigen ein sehr protoplasmareiches, körniges Epithel. Die Körnchen verschwinden auf Zusatz von Essigsäure.

\section{Versuch II.}

Einem kleinen, aber kräftigen grauen Kaninchen öffnete ich Morgens 8 Uhr 45 Min. die Bauchböble etwas nach links von der Linea alba, ohne Blutung, fing einige sich vordrängende Darmschlingen in einem feuchtwarmen Tuch auf und führte den Draht nach Spaltung des Peritoneum um die Arteria renalis sinistra. Die Operation verlief sehr schnell, war um $9 \mathrm{Uhr}$ vollendet. Das Thier erholte sich bald, frass mit gutem Appetit diesen und den folgenden Tag; war noch am Abend des zweiten Tages munter. Am Morgen des dritten Tages wurde es todt, jedoch noch nicht todtenstarr gefunden. Bauchwunde schon ziemlich fest verklebt. Därme vollkommen frei, selbst nicht an der Wonde adhärent. Nirgends Exsudat in der Bauchhöhle. Serosa glatt und spiegelnd, nicht injicirt. Dünndarm eng. Der Draht sass an der richtigen Stelle vor der Theilung der Arterie. Das Blut in der Arterie flüssig, über die stenosirte Stelle verschiebbar. Schon auf der Oberfầche bot die linke Niere ein trübes, gelblich-graues Aussehen dar, noch deutllcher dasselbe auf der Schnittfläche der Rindensubstanz, während die Marksubstanz, stark hyperämisch, sich scharf gegen die Rinde absetzte. Mikroskopisch fanden sich zahlreiche kleine Fettröpfchen, durch Essigsäurezusatz scharf vortretend, namentlich in der Umgebung der Kerne in grosser Zahl angehäuft, ohne zu confluiren, dies in der Rinde sowohl, als in der Marksubstanz. Das interstitielle Gewebe völlig normal. Die andere Niere zeigte keine Veränderungen.

\section{Versuch III.}

Die gleichen Verhältnisse, nur in weniger weit vorgeschrittenem Zustand, sah ich in der Niere eines anderen Kaninchens, welches in ähnlicher Weise, mit dem Bauchschnitt in der Linea alba operirt wurde, nach 24 Stundeu starb: deutliche Fetttröpfchen in der Rinde, wie in der Marksubstanz. Die andere Niere ohne Veränderungen.

\section{Versuch IV.}

Einem mittelgrossen, grauen Kaninchen lege ich eine Rückenwunde an, stülpe die linke Niere hervor und fange sie in einem feuchtwarmen Tuch auf: schlinge dann den Draht um die Arteria renalis. Die Niere hat anscheinend durch die 
Operation nicht gelitten. Das Thier ist in den folgenden Tagen durchaus munter: nach 4 Tagen tödte ich es. Wunde ohne Eiterung verbeilt, nirgends in der Tiefe Eiterung. Die Vena renalis intact. Die Arterie durchschneide ich und spritze peripher eine Lösung von Berliner-Blau unter geringem Druck, langsam ein: sofort dringt sie durch die verengerte Stelle hindurch. Auf der Oberfläche ist die Niere anämisch, dabei weisslich-trüb. Die trüben Stellen als Pänkttchen, Strichelchen ziemlich deutlich zn unterscheiden: nirgends necrotische Heerde. Ebenfalls ist die Sehnittfläche anämisch und trotz der Blässe trüber, weisslicher, als normal; aber nirgends von dem fahlen, glanzlosen Farbenton, der necrotischen Partien eigen. Ueberall tritt frisches Blut ans. Mikroskopisch in der Rinde, wie in der Marksubstanz die Epithelien der Harnkapälchen mit hellglänzenden Kügelchen erfülit, in grosser Zahi letztere, ohne zu confluiren. Sie verschwinden bei Extraction mit Alkohol und Aether, treten deutlich auf Zusatz von Essigsäure oder verdünnter Natronlauge hervor, während die übrigen Theile abblassen. Ausserdem finden sich auf jedem Schnitt der Rinde, wie der Marksubstanz Farbstoflkörnchen von BerlinerBlau, welche bis in die Niere vorgedrungen sind. Die andere Niere normal.

Betrachten wir nun die Bedeutung der beschriebenen Versuche, so ist es klar, dass wir durch die Verengerung der Nierenarterie, als einer Endarterie, eine permanente und im Ganzen wohl constante Verringerung der arteriellen Zufuhr mit Herabsetzung des arteriellen Druckes in dem betreffenden Organ erzielt haben. Die Folge ist eine Ernährungsstörung der Epithelien des Organs in Form der fettigen Degeneration. Nicht sieht man dies bei völliger Sistirung der arteriellen Zufuhr eintreten, sondern dann necrotisirt der betroffene Theil, und in necrotischen Partien habe ich keine Fettkörnchen gefunden. Dass später diese nicht mehr lebendigen Theile weitere chemische Umsetzungen erfahren, dass vielleicht auch Glieder der Fettgruppe dabei auftreten können, das ist wohl möglich: das sind aber Metamorphosen, weit verschieden von dem Prozess, um den es sich hier handelt, welcher ein vitaler und primärer ist. Es giebt aber noch eine dritte Ernährungsstörung, und sie beruht auch jedenfalls auf Verminderung der Ernährung: ich meine die einfache Atrophie, den regressiven Act, welcher dem physiologischen Entwickelungsgange am nächsten steht. Denn wie physiologisch im Alter, so kommt er uns pathologisch auch ja so vielfach vor, gleichsam als vorzeitige senile Involution, bei den chronischen, abzehrenden Krankheiten, zugleich mit einer allmählichen Verminderung der Blut- und Säftemasse. Experimentell ist dieser Prozess an der Niere gleichfalls leicht darzustellen. Nach Unterbindung des Ureter werden die Epithelien der gewundenen Harnkanälchen nicht 
lange Zeit hernach (ich sah es schon nach 8-10 Tagen bei gleichzeitigen Injectionen von harntreibenden Mitteln) kleiner und heller gefunden, verlieren mehr und mehr ihre Körnung, welche man wohl mit Recht als das anatomische Zeichen ihrer guten Function ansieht. Zugleich das ganze Organ blass und blutarm: und diesem Zustande folgt die Abnahme des Epithels. Denn nicht, glaube ich, kann man den Druck des gestauten Urins an und für sich als die Ursache ansehen, sondern er wirkt mit und durch die Anämie.

In diesem Experiment also sehen wir mit langsam zunehmender Anämie (oder, wetın man will, Druck) Hand in Hand gehend, eine Rückbildung der zugebörigen Organbestandtheile: fettige Degeneration sahen wir schnell eintreten, auf eine Ursache hin, welche mehr schnell und direct den Ernährungsvorgang beeinflussen musste. Und hiermit stimmen auch in ibrer Erscheinungsweise überein manche wohlgekannte Thatsachen aus der menschlichen Physiologie und Pathologie.

Ich erinnere z. B. an die fettige Degeneration des Uterus im Puerperium: dann an die fettigen Degenerationen, welche entzündliche Zustände begleiten. Zwar ist es, mit letzteren vorzugehen, immerhin etwas misslich: denn uns geht die wahre Erkenntniss über das Zustandekommen der Ernährungsstörung hierbei ab, wenn wir auch die Coincidenz beider Zustände fast stets constatiren, zumal bei den acuten Entzündungen, z. B. bei der acuten Nephritis.

Unklarer noch liegen die Verhältnisse für die chronischen, proliferirenden. Der chronische Morbus Brighti geht bald mit fettiger Entartung, bald fast ohne solche, mit einfacher Atrophie einher. Wann, das ist wohl noch nicht ganz entschieden. Möglich ist es und leicht denkhar, dass auch hierbei die Intensität der Entzündung entscheidend ist, dass es sich verhält vielleicht, wie mit den phthisischen Zuständen, welche auch in allen Graden, vom ausgedehntesten Zerfall bis zur einfachen Vernarbung herab, sich darstellen können. Berücksichtigen wir hierbei noch, dass man für die rasch zerfallenden phthisischen Prozesse entzündliche Zustände der zuführenden Arterien mit Obliteration derselben in der lezten Zeit als Ursache aufgestellt hat ${ }^{\mathbf{y}}$ ).

Doch es giebt ja in der menschlichen Pathologie auch sehr

1) Köster, Friedländer. 
elementare, beinahe experimentelle Prozesse, welche mit Ernährungsstörungen einhergeben: die Thrombose und die Embolie.

Von ihnen müssen auch Aufschlüsse über das Zustandekommen von fettiger Degeneration zu erwarten sein. So einfach der Prozess der Enbolie aber auch scheint, so ist man doch auch hier, glaube ich, zu einem Abschluss in Betreff der Schicksale des betroffenen Theiles noch nicht gelangt. Ich halte mich im Folgenden speciell an die Niereninfarcte.

Durch die Experimente von Cohnheim ist es bekannt geworden und wieder und wieder von anderen Sejten bestätigt, dass die Niere nach Sistirung des arteriellen Zuflusses nicht der fettigen Degeneration, sondern der Necrose anheimfällt. Auch ein Beispiel wahrer Embolie, beim Menschen beobachtet, liegt vor ${ }^{1}$ ). Nun ist die Nierenarterie eine Endarterie: ebenso aber auch ihre Aeste. Man sollte von ihrer Verlegung also ein gleiches Resultat erwarten. Dennoch findet man in den Handbüchern der pathologischen Anatomie (Klebs, Förster) angegeben, dass bei Embolie eines Astes die zugehörigen Theile des betroffenen Bezirkes der fettigen Degeneration unterliegen, Mir fehlt es an einem hinreichend grossen Material, um diese Sache im Augenblick genügend zu prüfen: und habe jch mich dem Thierexperiment deshalb zugewandt, habe einem Kaninchen einen Ast der Nierenarterie unterbunden.

\section{Experiment $V$.}

Nach 28 Stunden war das Thier gestorben. In der Niere zeigte sich der betroffene Bezirk scharf gegen das übrige Parenchym durch seine Färbung abgesetzt. Er war in hohem Grade trüb, gelb, lehmfarben gefärbt, doch nicht so ganz gleichmässig, dass man bei genauer Betrachtung nicht schon makroskopisch gewisse Unterschiede zwischen einem ovalen centralen gelbbraunen Fleck und dem umgebenden, an das Gesunde grenzenden Theile hätte wahrnehmen können. Ganz in der Nähe der Grenze gegen das Gesunde, parallel mit ihr, aber nicht in ihr lief ein dunkelrother Streif. Auf der Schnittfläche bot die Rindensubstanz ein analoges Bild: die Marksubstanz war nicht durchweg gelb verfärbt, sondern in ihr natm der dunkelrothe Streif an Breite zu und batte in der unteren Hälfte allein das Terrain occupirt. Mikroskopisch zeigten die centralen Partien der betroffenen Rindensubstanz ein sehr trübes Epitbel, so dass die Kerne kaum sichtbar waren. Die Trübung war eine sehr feinkörnige, hellte sich bei Zusatz von Essigsäure beträchtlich, bei Zusatz von verdünnter Natronlange gänzlich auf, war also sicher kein Fett. Dagegen traf man in der peripheren Zone viele hellglänzende Kügelchen, oft sogar von relativ beträchtlicher

1) Cohnheim, Embol, Prozesse. S. 77. 
Grösse. Sie lagen im interstitiellen Gewebe und in den Epithelien der Harnkanälchen, doch so, dass an der Grenze gegen das Gesunde vorwiegend die Harnkanälchen, weiter nach innen nur die Zellen der Glomeruli und des interstitiellen Gewebes der Degeneration anheimgefallen waren, während das zugehörige Epithel schon fast durchweg den Zustand der Necrose erkennen liess. Unmittelbar unter der Kapsel fand ich die nächsten Harnkanälchen, auch innerhalb der necrotischen Partien, mit Fettkőrnchen erfüllt. Das Blut an der Grenze gegen daş Gesunde hin in frischem Zustand, in dem erwähnten rothen Streif in beträchtlicher Menge vorhanden. Die Marksubstanz zeigte die Capillaren strotzend gefüllt, dazwischen die Epithelien in körnigem Zerfall. Die nicht betroffenen Partien der Niere sind völlig normal.

Sicher lag in diesem Falle die Vermuthung nahe, dass die Kügelchen entfärbte Blutkörperchen darstellen möchten: und trotz ihrer Resistenz gegen Essigsäure und verdünnte Natronlauge, wäre ich mit meinem Urtheil sehr vorsichtig gewesen, wenn sie nicht auch bei Extraction mit Alkohol und Aether verschwunden wären.

Sehe ich mich nun in der Literatur um, so finde ich aus der menschlichen Pathologie Fälle erwähnt, welche Aehnliches constatiren: so ein Fall von hämorrhagischem Niereninfarct, bei Thrombose eines Astes der Nierenarterie, veröffentlicht von Recklinghausen ${ }^{1}$ ):

„Der grösste Theil des Infarctes hatte cine gleichmässige, intensiv weisse Farbe, nur eine 1 Linie breite Mantelschicht war grüngelblich gefärbt. Nach aussen von letzterer folgte eine etwas breitere, sehr schlafie, stark gerötbete Zone, welche an der Nierenoberfläche sich als flache Einsenkung markirte....... Im Gegensatz hierzu waren Harnkanälchen und Glomeruli innerhalb des Infarcts von ziemlich normaler Grösse, erstere gefüllt mit einer feinkörnigen, auf Zusatz von Essigsäure lichter werdenden Masse mit sehr undeutlichen Kernen. . . . A Auffallend contrastirte mit diesen blassen Partien die erwähnte gelbe Mantelschicht, welche der dichten Einlagerung von meist sehr grossen Fetttropfen ein gleichmässig schwarzes Aussehen unter dem Mikroskop verdankte. Nach innen zu wurde diese Schicht allmăhlich lichter, und hier konnte man sehr dentlich eine Anordnung der Fettropfen nach den Interstitien erkennen, während die Harnkanälchen dieselbe blasse Beschaffenheit hatten, wie in den centralen Theilen des Infarcts" etc. Recklinghausen hält die Fettablagerung für eine Infiltration.

7) Dieses Archiv Bd. 20. S. 205. 
Ein ganz ähnliches Bild erhielt ich bei einem Kaninchen, welches durch Verengerung eines Astes der Nierenarterie eine Thrombose desselben erlitten, nach 6 Tagen getödtet wurde: In den peripheren, etwas gegen das Gesunde eingesunkenen Partien von trüber, hellgelblicher Färbung viele Fettkörnchen in den Epithelien der Tubuli contorti nebst geringer Wucherung im interstitiellen Gewebe weiter nach innen wird die Färbung gelb, und darin die Epithelien necrotisch, nur die Zellen des interstitiellen Gewebes in fettigem Zerfall begriffen, sowie sebr schön fettige Degeneration in einer arteriellen Gefässwand.

Ich könnte ferner aus der Arbeit von Beckmann, „über hämorrbagische Infarcte der Nieren " ${ }^{1}$ ) einige Falle von ausgedehnter Embolie (z. B. Fall 6 u. 7) citiren mit ähnlichen Erscheinungen: „die Kanäle sind trübe .... in dem Stroma fast überall liegen feinere und gröbere Fetttröpfchen" "2). Aber im Ganzen, wie gesagt, findet man die Meinung, dass nach stattgehabter Embolie die Theile der fettigen Degeneration anheimfallen. Wie lässt sich dies mit meinem Experiment und den mitgetheilten Befunden vereinigen? Zunächst handelt es sich in meinem Experiment um einen grossen Heerd, welcher durch Rinde und Marksubstanz ging: bei kleinen mag in der That nur fettige Degeneration zu finden sein. Es ist dies aber kein Gegenbeweis. Denn wenn man nach der Ursache der fettigen Degeneration der Randpartien fragt im Gegensatz zur centralen Necrose, so unterliegt es für mich keinem Zweifel, dass erstere einer Ernährung von Seiten aes gesunden Gewebes ibre Entstehung verdankt, den collateralen Verbindungen von Capillaren unter einander. So entsteht noch ein Blut- und Saftstrom in den Randpartien des Infarctes, obschon nicht ausreichend zur Integrität. Folge ist fettige Degeneration. In den weiter abgelegenen Partien muss

1) Dieses Archiv Bd. 20. S. 217.

2) Ein frisch untersuchter Niereninfarct von anscheinend nicht langem Bestand bot mir Gelegenheit, Gleiches zu constatiren: in der Peripherie des Infarcts, in der hyperämischen Zone und nächster Umgebung, reichliche Fettkö̀nchen, auch im Verlauf der Harnkanälchen. Weiterhin wird die Lagerung derselben netzförmig d. b. interstitiell zwischen den durch $\mathrm{NaOH}$ abblassenden Harnkanälchen, bis auch sie mehr und mehr gegen das Centrum hin schwindet. Von der Spitze des Keils ragt ein rother Streif weit in das Centrum des Infurcts hinein: die verstopfte Arterie. 
natürlich die Strömung mehr und mehr sistiren: früber für dje Epithelien der Harnkanälchen, später für das interstitielle Gewebe. Bei kleinen Heerden aber bleibt es ja immer möglich, dass eine geringe Blutströmung im ganzen Bezirk des verstopften Gefässes unterhalten werden kann (s. Beckmann l. c. S. 225: „Für gewöhnlich nun stellt sich der Kreislauf soweit her, dass ein geringes Fortleben des ergriffenen Theiles möglich ist; es tritt dann, wie gewöhnlich, Fettmetamorphose auf").

Dazu kommt noch, und das ist wohl zu berucksichtigen, dass Unterbindung und Embolie nicht wohl ganz dieselben Dinge sind. Eine Embolisirung durch losgerissene Thrombenstücke braucht ja überhaupl nicht sogleich das Lumen des Gefässes vollständig auszufüllen; sicher geht oft noch zunächst ein geringer Blutstrom hindurch, vielleicht gar nicht so kurze Zeit, bis endlich weitere thrombotische Massen den Weg ganz verlegen, oder eine weitere Einkeilung des losgerissenen Stückes in kleinere Aeste erfolgt.

Auch verschliessen wir bei der Unterbindung Gefässe, welche bei Embolie wegsam bleiben, nehmlich die Gefässe der Gefässwand selber: und sie mögen immerhin, gerade in der Gefässswand und ihrer Umgebung, einen geringen Blutlauf unterhalten können.

Es bleibt jetzt für die experimentellen Untersuchungen der Girculationsanomalien in der Niere und ihrer Bedeutung für das Parenchym noch eine Frage übrig, welches die Wirkung der venösen Stauung bei gleichzeitjger Anämie sein wird: und dies ist ja eigentlich das Verhältniss bei der richtigen Nierenstauung der Herzkranken u. s. w. Darüber hoffe ich in der nächsten Zeit weitere Mittheilungen machen zu können. 